\title{
Orthopedics and COVID-19: Scientific Publications Rush
}

\author{
Riccardo D'Ambrosi ${ }^{1}$ (1)
}

Received: 24 April 2020 / Accepted: 7 May 2020 / Published online: 25 May 2020

(c) Indian Orthopaedics Association 2020

\begin{abstract}
Background The purpose of this literature review is to analyze all the articles related to COVID-19 and orthopedics and to shed light on the scientific evidence that has actually been found.

Methods A systematic review of the existing literature was performed to identify all studies dealing with COVID-19 and orthopedics. The guidelines of the Preferred Reporting Items for Systematic Reviews and Meta-analyses (PRISMA) were followed for the identification of the articles. On April 19 2020, a search was performed using MEDLINE and PubMed Central. For the research, the following terms were used: "COVID-19" OR "SARS-CoV-2" OR "Coronavirus" AND "orthopaedic" OR "orthopedic" OR "traumatology".

Results A total of 23 studies were included in the review, of which 19 (82.6\%) were editorials, letters to editors, orthopedic forums or expert opinions, 3 (13.0\%) studies were level IV of evidence and 1 (4.4\%) was a literature review. Articles have been divided into the follow categories: guidelines, traumatology, pediatric, spine surgery, telemedicine, sports medicine, bioethical, educational, rehabilitation, survey and hand surgery.

Conclusions Although scientific production is very active on the topic of COVID-19 and orthopedics, the scientific evidence is practically nil, mainly dealing with editorials, letters to editors or expert opinions. Therefore, future studies should focus on clinical outcomes and treatments in COVID-19-positive orthopedic patients and prevention strategies. Additionally, international guidelines and consensus statements must be developed to standardize procedures as much as possible in this pandemic scenario.
\end{abstract}

Keywords COVID-19 $\cdot$ SARS-CoV-2 $\cdot$ Pandemic $\cdot$ Literature review $\cdot$ Scientific publications

\section{Introduction}

In December 2019, a new kind of pneumonia disease was recognized in Wuhan, the People's Republic of China. The outbreak was declared a Public Health Emergency of International Concern on January 30 2020, and the World Health Organization (WHO) announced a name for this virus [SARS (severe acute respiratory syndrome)-CoV-2] and for the disease (COVID-19) on February 11 2020, and formally declared it a pandemic one month later on March 112020 [1].

The coronaviruses (CoVs) are considered to be the major pathogens behind emerging respiratory disease outbreaks. They are a large family of single-stranded RNA viruses that

Riccardo D'Ambrosi

riccardo.dambrosi@hotmail.it

1 IRCCS Istituto Ortopedico Galeazzi, Via Galeazzi 4, 20161 Milan, Italy can be isolated in different animal species. Moreover, for reasons yet to be explained, these viruses can cross species barriers and can cause, in humans, illnesses ranging from the common cold to more severe diseases such as MERS and SARS [2].

The emergence of the COVID-19 pandemic has severely impacted medical practices throughout the world, especially those of infectious disease specialists, emergency room personnel, anesthetists, intensivists, virologists, epidemiologists and hygienists. At the same time, researchers have also been affected. The majority of non-essential clinical staff have been sent home to work remotely, thereby providing opportunities for increased efforts focused on scientific research [3].

Just in the context of COVID-19, an increase in scientific articles on the topic has been noted in recent weeks; this has also been facilitated by the fact that more than 30 leading publishers have committed to making all their COVID-19 and coronavirus-related publications_-and the available data 
supporting them-immediately accessible on PubMed Central (PMC) and other public repositories [4]. This will help to support the ongoing public health emergency response efforts.

The purpose of this literature review is to analyze all the existing articles related to COVID-19 and orthopedics, and to shed light on what scientific evidence has actually been found.

\section{Methods}

A systematic review of the existing literature was performed to identify all studies dealing with COVID-19 and orthopedics. The PRISMA guidelines were followed for the identification of the articles. On April 19 2020, a research was performed using MEDLINE and PubMed Central [5]. For the research, the following terms were used: "COVID-19" OR "SARS-CoV-2" OR "Coronavirus" AND "orthopaedic" OR "orthopedic" OR "traumatology". The research also included the references of all the articles identified and the references of the systematic review. At the first screening, we identified 57 articles, of which we selected 23 that satisfied our inclusion criteria (Fig. 1). Considering the heterogeneity of the articles, they were, therefore, divided by category according to the topic discussed. All types of studies according to "The Oxford 2011 Levels of Evidence" were included in this review [6].

\section{Results}

A total of 23 [7-29] studies were included in the review, of which 19 [7-9, 11-18, 20-27] (82.6\%) were editorials, letters to editors, orthopedic forums or expert opinions, 3 $[19,28,29]$ studies were level IV (13.0\%) of evidence and 1 [10] was a literature review (4.4\%). Articles have been divided in the follow category: guidelines, traumatology, pediatric, spine surgery, telemedicine, sports medicine, bioethical, educational, rehabilitation, survey and hand surgery.
Fig. 1 A flowchart of the literature screemimg performed in this study

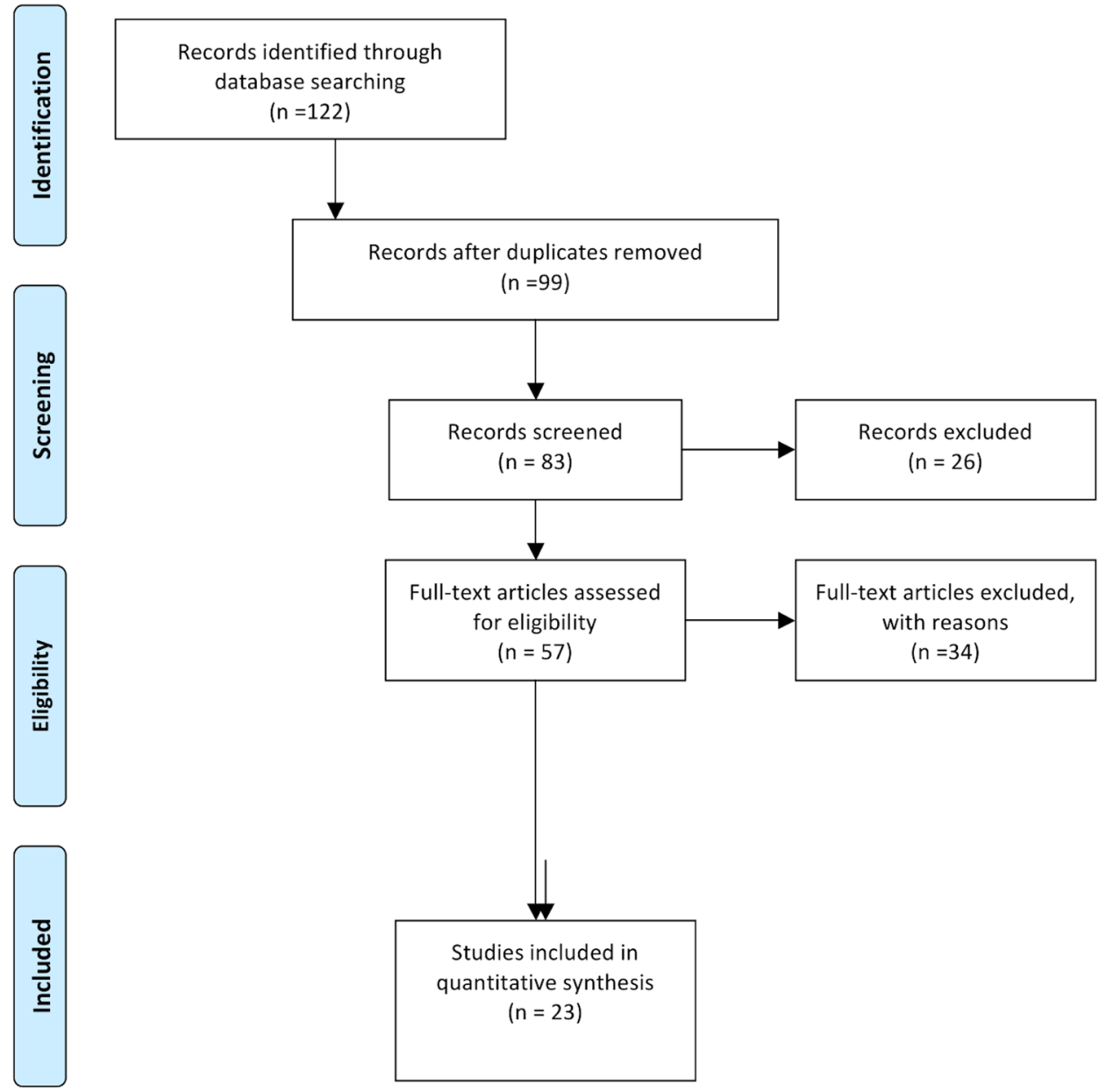




\section{Guidelines}

Sarac et al. analyzed current guidelines published by each state in the USA, concluding that few states have published guidelines specific to orthopedic surgery during the COVID19 outbreak, leaving hospital systems and surgeons with the responsibility of balancing the benefits of surgery with the risks to public health [7].

DePhilippo et al. outlined general guidelines for surgical procedures that ought to be considered necessary in the case of:

- Acute and/or disabling injuries to health-care workers, first responders, and members of the military and police and fire departments;

- Osseous fractures and/or irreducible joint dislocations; and

- Wound/joint infections or postoperative wound dehiscence.

Furthermore, the authors stratified surgical procedures according to the joints in which surgical necessity ought to be considered, in case of pathologies that could lead to long-term disability and chronic pain if acute surgical management is delayed [8].

Mauffrey and Trompeter published a long editorial reporting several issues linked with COVID-19, in particular by addressing the following topics: clinical care and how it is delivered, communication, education and meetings, research, operations, and ethical considerations [9].

Massey et al. considered it crucial for orthopaedic departments to remain flexible and accommodating in light of the pandemic's perpetual evolution and its consequential healthcare implications. Modifying surgical selection is paramount to fulfilling the needs of the entire health system. Depending on the needs of the local community and the phase of COVID-19 outbreak in a specific area, the surgical selection priorities may change. It remains important that orthopedic programs emphasize personnel safety and slowing the spread of the virus so that the department can still maintain vital functions. Emerging technologies such as inpatient telemedicine and online file sharing applications can enable orthopedic programs to still function, while attempting to protect medical staff and patients from the COVID-19 spread [10].

Awad et al. provided evidence-based recommendations and measures for the appropriate personal protective equipment for different clinical and surgical activities in various settings. To reduce the occupational risk in treating suspected or confirmed COVID-19 orthopedic patients requiring urgent care, recommended precautions and preventive actions (to applied in the triage area, emergency department consultation room, induction room, operating room, and recovery room) should be reviewed [11].
Vaccaro et al., representing some of the larger musculoskeletal multispecialty groups in the country, provided a framework with recommendations to best weather the ensuing months. These recommendations allow providers and their staff to return to an infrastructure that can adjust immediately to the pent-up healthcare demand that may occur following the COVID-19 pandemic. In the editorial suggestion, the authors reported about practice finances, staffing, telehealth, operational plans following the crisis and ethical considerations [12].

Valshya et Valsh reviewed the current guidelines already published in the literature and also reported the Royal College of Surgeons of England's guidelines for good practice. The authors' take-home message is as follows:

"It is important to realize that these healthcare workers are the ones which are on the frontline in this war against the deadly virus and must be supported whole heartedly by the society and the governments, in this time of crisis. They must be provided adequate numbers of good-quality personal protective equipment (PPEs) and insurance cover, to be able fight in this battle for us all. There has been an acute shortage of these essential PPEs for them and providing them with substandard PPEs (similar to rain coats!) is not the solution to overcome the crisis. It is akin to 'sending soldiers into the war with misfiring guns" [13].

The editorial by Sarpong et al. is a return to the past in which the figure of the orthopedist is re-defined in light of orthopedic surgeons being asked to perform forgotten procedures changing the normal clinical activity [14].

In consideration of the extraordinary event we are victims of, Halawi et al. analyze how normal clinical and surgical activity must change, suggesting new guidelines ad hoc for pandemic [15].

\section{Traumatology}

Stinner et al. emphasize how it is important to optimize medical resource allocation and prevent the spread of SARS-CoV-2, and how, to this end, orthopedic trauma surgeons must remain vigilant and be prepared to optimally care for the injured trauma patient [22]. Furthermore, they reported three clinical cases demonstrating a change in practice to minimize patient morbidity and potential spread of SARS-CoV-2".

Rodrigues-Pinto et al. underline the importance of the protection of health workers and how appropriate training is of paramount importance to minimizing their infection and the stress associated with the physical and mental exhaustion they have to endure. The correct application of these procedures is expected to adequately protect health workers by giving them access to protective clothing readily available at most operating rooms, thereby minimizing the risks associated with the lack of personal protective equipment [21]. 
Mi et al. summarized the clinical characteristics and early prognosis of COVID-19 infection in a cohort of ten patients with fractures; these tended to be more severe than those reported for adult patients with COVID-19 without fracture. This finding may be related to the duration between the development of symptoms and presentation. Surgical treatment should be carried out cautiously or non-operative care should be chosen for patients with fracture in COVID-19-affected areas, especially for older individuals with intertrochanteric fractures [19].

Ashford et al. broadly analyzed the situation of COVID19 and the role of the orthopedic surgeon, in particular focusing on 4 key points:

- PPE (personal protective equipment);

- Avoid (avoid contact, avoid transfer of patients, avoid surgery);

- Restrict (restrict number of visits, restrict generation of aerosols, restrict visitors); and

- Abbreviate (abbreviate waiting times, abbreviate treatment) [20]

\section{Pediatric}

Farrell et al. published guidelines based on expert opinion and best available evidence, providing a framework for the management of pediatric orthopaedic patients during the COVID pandemic. The general principles include limiting procedures to urgent cases such as traumatic injuries and deferring outpatient visits during the acute phase of the pandemic. Moreover, non-operative methods should be considered where possible. For patients with developmental or chronic orthopaedic conditions, it may be possible to delay treatment for 2-4 months without substantial detrimental long-term impact [16].

\section{Spine Surgery}

Donnally III et al. reported some key points to consider in spine surgery during COVID-19:

- Importance of daily's updates from the hospital.

- The urgency of surgical intervention for each patient being agreed upon by members of the spine department.

- Avoiding the assumption that surgical delays will be for a short duration.

- Considering alternative surgical plans and less invasive amoptions depending on hospital bed availabilityfor spine surgeries that cannot be postponed [17].

\section{Sports Medicine}

Corsini et al. analyzed the possible effects of coronavirus on athletes, particularly on the resumption of football activity, reccomending maximal caution to the football governance on the decision regarding when to restart sport activity. Moreover, a specific protocol should be considered to check cardiological, pulmonary, and, in general, systemic sequelae of COVID-19 before resuming sporting activities [23].

Gilat and Cole published an editorial recalling the importance of sport and consequently the role of sports medicine. Accordingly, health-care providers specializing in sports medicine who attend to all types of athletes must quickly adapt to this new and hopefully transient situation continuing to serve their community to the best of their ability. Furthermore, efforts should be made to maximize the use of "telemedicine" to continue providing care to patients and identify essential problems that require observation in the clinic: a physical examination, imaging for definitive assessment, emergency department triage, and defining and treating conditions that require urgent and time-sensitive surgical care. Staff should be stringent with their own protection at all times, as they can easily become "super-spreaders," infecting whole communities [24].

\section{Telemedicine}

Loeb et al. reported the difficulties in the direct relationship with the patient during the pandemic, analyzing how the use of telemedicine can change these scenarios. The authors report the experience in unit care where a robust telemedicine program during a 5-day period was introduced. Between telemedicine encounters and necessary in-person visits, providers may be able to achieve $50 \%$ of their typical clinic volume within 2 weeks. Further, when handling the massive disruption to the routine patient care workflow, it is critical to understand the key factors associated with an accelerated introduction of telemedicine for the safe and effective continuation of orthopaedic care during this pandemic [18].

\section{Bioethical}

Dunham et al. provided a framework for considering how a global pandemic changes our moral responsibilities. Pandemic environments, however, bring challenges that can only be addressed by moving to a public health ethics frame. The standard question of my actions at this time is modified to what we, as a population, ought to do. The authors highlight a disconnection between one's pre-COVID-19 sense of 
responsibility and the one that is developing now; disconnect is ripe for moral distress, residue, and injury [25].

\section{Educational}

Kogan et al. have assessed the appropriateness of educational tools in the form of web-based learning, surgical simulators, and basic competency tests to help programs ensure continued resident growth, development, and wellbeing, while maintaining high-quality patient care. The authors conclude that orthopaedic departments must develop a list of priorities based on their institutional needs to guide decision-making during these times of uncertainty. In this scenario, residents' roles should be redefined, in particular, changing the perspective on learning methods. New learing tools include the use of virtual meeting platforms, independent home study, surgical simulation, and fostering leadership opportunities. Furthermore, it is mandatory for the residents to feel safe, protected, and heard [26].

\section{Rehabilitation}

On March 13th, the Italian Society of Physical and Rehabilitation Medicine decided to produce a short document with the aim of providing recommendations fully compliant with the national and regional provisions, but specifically focused upon the rehabilitation sector, to support the physical and rehabilitation medicine physicians, other medical specialists, other health professionals, and policy markers in taking decisions during an unusual and largely unknown circumstance [27].

\section{Survey}

Guo et al. conducted a survey to identify the orthopaedic surgeons infected with COVID-19 in Wuhan. Social demographic variables, clinical manifestations, exposure history, awareness of the outbreak, infection control training provided by hospitals, and individual protection practices were analyzed throgh the questionnaire. Furthermore, the possible risk factors at the individual level—a 1:2 matched case-control study - was observed. A total of 26 orthopaedic surgeons from 8 hospitals in Wuhan were identified as having COVID-19. The incidence in each hospital varied from $1.5 \%$ to $20.7 \%$. The onset of symptoms was from January 13 to February 5, 2020, peaking on January 23, 8 days prior to the peak of the public epidemic. The suspected sites of exposure were general wards (79.2\%), public places at the hospital $(20.8 \%)$, operating rooms $(12.5 \%)$, the intensive care unit (4.2\%), and the outpatient clinic (4.2\%). There was transmission from these doctors to others in $25 \%$ of cases, including to family members $(20.8 \%)$, colleagues $(4.2 \%)$, patients (4.2\%), and friends (4.2\%). Moreover, participation in real-time training on prevention measures was found to have a protective effect against COVID-19. Furthermore, not wearing an $\mathrm{N} 95$ respirator was found to be a risk factor and wearing respirators or masks all the time was found to be protective. Severe fatigue was found to be a risk factor for infection with COVID-19 [28].

\section{Hand Surgery}

Ducornau et al. described the different measures used in response to the COVID-19 pandemic by hand surgeons all over the world, comprising 47 surgeons working in 34 countries. They found several protocols exist in terms of visitors, health professionals in the operating room, patient waiting areas, wards, and emergency rooms [29].

\section{Discussion}

The main findings of the current review primarily concern the high number of published studies concerning COVID-19 and orthopedics in such a short time; in fact, the first article on a temporal level dates back to March 16, thus showing an average of almost one article per day [27].

At the same time, however, almost all published articles have a very low scientific value (editorial, expert opinion) and only 3 studies have scientific evidence (Level of Evidence-4) [6].

This shows how all the efforts we are carrying out on COVID-19 in orthopedics are empirical, without a demonstrated and demonstrable scientific value and how numerous articles still have to be published on the topic, reporting clinical data, results about treatment, and prevention strategies.

In particular, it is possible in the current review to see how several articles trace the guidelines at hospital and surgical and outpatient level in the COVID-19 era, but many open questions still remain: how should patients with orthopedic and COVID-19 diseases be treated?

Moreover, do the traumatology emergency patients COVID-19 positive deserve a different anesthesiological and pharmacological rehabilitation treatment?

Furthermore, these clinical results will have to be evaluated over time to understand if the employed measures were correct without affecting health and quality of life; these observations will not only be of orthopedic relevance but also must concern infectious disease specialists, pulmonologists, and anesthesiologists.

Future studies will, therefore, have to create "gold standard" treatment, with international and multi-disciplinary guidelines in patients with COVID-19 through the publication of scientific papers of the highest level of evidence possible. 
Finally, almost all the articles relating to the guidelines highlighted the importance of telemedicine, avoiding or reducing contacts as much as possible, and safeguards; none of these articles, however, analyzed how to behave when we will be able to return to normal activities.

Consequently, several questions remain unclear:

- When will normal clinical activity be possible?

- How will it be developed?

- Will we be able to have COVD-19 free structures for orthopedic patients?

There is still no answer to these questions, thereby leaving enormous doubts open and the certainty that after this pandemic, nothing will resemble the past in both daily and working life.

\section{Conclusions}

Although scientific production is very active on the topic of COVID-19 and orthopedics, the scientific evidence is practically nil, mainly dealing with editorials, letters to editors or expert opinions. Therefore, future studies should focus on clinical outcomes and treatments in COVID-19-positive orthopedic patients and prevention strategies. Additionally, international guidelines and consensus statements must be developed to standardize procedures as much as possible in this pandemic scenario.

Funding None.

\section{Compliance with Ethical Standards}

Conflict of interest The authors declare that they have no conflict of interest.

Ethical standard statement This article does not contain any studies with human participants or animals performed by any of the authors.

Informed consent For this type of study, formal consent is not required.

\section{References}

1. Rothan, H. A., \& Byrareddy, S. N. (2020). The epidemiology and pathogenesis of coronavirus disease (COVID-19) outbreak. Journal of Autoimmunity, 109, 102433.

2. Lai, C. C., Shih, T. P., Ko, W. C., Tang, H. J., \& Hsueh, P. R. (2020). Severe acute respiratory syndrome coronavirus 2 (SARSCoV-2) and coronavirus disease-2019 (COVID-19): The epidemic and the challenges. International Journal of Antimicrobial Agents, 55(3), 105924.
3. Fiorillo, A., \& Gorwood, P. (2020). The consequences of the COVID-19 pandemic on mental health and implications for clinical practice. European Psychiatry, 63(1), e32.

4. https://www.ncbi.nlm.nih.gov/pmc/about/covid-19/. Accessed 19 Apr 2020.

5. Moher, D., Shamseer, L., Clarke, M., Ghersi, D., Liberati, A., Petticrew, M., et al. (2015statement). Preferred reporting items for systematic review and meta-analysis protocols (PRISMA-P) 2015statement. Systematic Review, 4, 1.

6. Marx, R. G., Wilson, S. M., \& Swiontkowski, M. F. (2015). Updating the assignment of levels of evidence. Journal of Bone and Joint Surgery, 97(1), 1-2.

7. Sarac, N. J., Sarac, B. A., Schoenbrunner, A. R., Janis, J. E., Harrison, R. K., Phieffer, L. S., et al. (2020). A review of state guidelines for elective orthopaedic procedures during the COVID19 outbreak. Journal of Bone and Joint Surgery. https://doi. org/10.2106/JBJS.20.00510.

8. DePhillipo, N. N., Larson, C. M., O'Neill, O. R., \& LaPrade, R. F. (2020). Guidelines for ambulatory surgery centers for the care of surgically necessary/time-sensitiveorthopaedic cases during the COVID-19 pandemic. Journal of Bone and Joint Surgery. https:// doi.org/10.2106/JBJS.20.00489.

9. Mauffrey, C., \& Trompeter, A. (2020). Lead the way or leave the way: leading a Department of Orthopedics through the COVID-19 pandemic. European Journal of Orthopaedic Surgery and Traumatology. https://doi.org/10.1007/s00590-020-02670-x.

10. Massey, P. A., McClary, K., Zhang, A. S., Savoie, F. H., \& Barton, R. S. (2020). Orthopaedic surgical selection and inpatient paradigms during the coronavirus COVID-19 pandemic. Journal of American Academy of Orthopaedic Surgeons. https://doi. org/10.5435/JAAOS-D-20-00360.

11. Awad, M. E., Rumley, J. C. L., Vazquez, J. A., \& Devine, J. G. (2020). Peri-operative Considerations in urgent surgical care of suspected and confirmed COVID-19 orthopedic patients: operating rooms protocols and recommendations in the current COVID19 pandemic. Journal of American Academy of Orthopaedic Surgeons. https://doi.org/10.5435/JAAOS-D-20-00227.

12. Vaccaro, A. R., Getz, C. L., Cohen, B. E., Cole, B. J., \& Donnally, C. J., 3rd. (2020). Practice management during the COVID-19 pandemic. Journal of American Academy of Orthopaedic Surgeons. https://doi.org/10.5435/JAAOS-D-20-00379.

13. Vaishya, R., \& Vaish, A. (2020). Roles and responsibilities of the orthopaedic community and the society during COVID-19 pandemic. Indian Journal of Orthopedics. https://doi.org/10.1007/ s43465-020-00105-7.

14. Sarpong, N. O., Forrester, L. A., \& Levine, W. N. (2020). What's important: redeployment of the orthopaedic surgeon during the COVID-19 pandemic: perspectives from the trenches. Journal of Bone and Joint Surgery. https://doi.org/10.2106/JBJS.20.00574.

15. Halawi, M. J., Wang, D. D., \& Hunt, T. R., 3rd. (2020). What's important: weathering the covid-19 crisis: time for leadership, vigilance, and unity. Journal of Bone and Joint Surgery. https:// doi.org/10.2106/JBJS.20.00419.

16. Farrell, S., Schaeffer, E. K., \& Mulpuri, K. (2020). Recommendations for the care of pediatric orthopedic patients during the COVID pandemic. Journal of American Academy of Orthopaedic Surgeons. https://doi.org/10.5435/JAAOS-D-20-00391.

17. Donnally, C. J., Shenoy, K., Vaccaro, A. R., Schroeder, G. D., \& Kepler, C. K. (2020). Triaging spine surgery in the COVID-19 era. Clinical Spine Surgery. https://doi.org/10.1097/BSD.00000 00000000988

18. Loeb, A. E., Rao, S. S., Ficke, J. R., Morris, C. D., \& RileyLevin, L. H. A. S. (2020). Departmental experience and lessons learned with accelerated introduction of telemedicine during the COVID19 crisis. Journal of American Academy of Orthopaedic Surgeons. https://doi.org/10.5435/JAAOS-D-20-00380. 
19. Mi, B., Chen, L., Xiong, Y., Xue, H., Zhou, W., \& Liu, G. (2020). Characteristics and early prognosis of COVID-19 infection in fracture patients. Journal of Bone and Joint Surgery. https://doi. org/10.2106/JBJS.20.00390.

20. Ashford, R. U., Nichols, J. S., \& Mangwani, J. (2020). Annotation: the COVID-19 pandemic and clinical orthopaedic and trauma surgery. Journal of Clinical Orthopaedics and Trauma. https://doi. org/10.1016/j.jcot.2020.04.002.

21. Rodrigues-Pinto, R., Sousa, R., \& Oliveira, A. (2020). Preparing to perform trauma and orthopaedic surgery on patients with COVID-19. Journal of Bone and Joint Surgery. https://doi. org/10.2106/JBJS.20.00454.

22. Stinner, D. J., Lebrun, C., Hsu, J. R., Jahangir, A. A., \& Mir, H. R. (2020). The orthopaedic trauma service and COVID-19 practice considerations to optimize outcomesand limit exposure. Journal of Orthopaedic Trauma. https://doi.org/10.1097/BOT.0000000000 001782.

23. Corsini, A., Bisciotti, G. N., Eirale, C., \& Volpi, P. (2020). Football cannot restart soon during the COVID-19 emergency! A critical perspective from the Italian experience and a call for action. British Journal of Sports Medicine. https://doi.org/10.1136/bjspo rts-2020-102306.

24. Gilat, R., \& Cole, B. J. (2020). COVID-19, medicine, and sports. Arthroscopy Sports and Medicine and Rehabilitation. https://doi. org/10.1016/j.asmr.2020.04.003.

25. Dunham, A. M., Rieder, T. N., \& Humbyrd, C. J. (2020). A bioethical perspective for navigating moral dilemmas amidst the
COVID-19 pandemic. Journal of American Academy of Orthopaedic Surgeons. https://doi.org/10.5435/JAAOS-D-20-00371.

26. Kogan, M., Klein, S. E., Hannon, C. P., \& Nolte, M. T. (2020). Orthopaedic education during the COVID-19 pandemic. Journal of American Academy of Orthopaedic Surgeons. https://doi. org/10.5435/JAAOS-D-20-00292.

27. Boldrini, P., Bernetti, A., Fiore, P., \& SIMFER Executive Committee, and SIMFER Committee for international affairs. (2020). Impact of COVID-19 outbreak on rehabilitation services and Physical and Rehabilitation Medicine (PRM) physicians' activities in Italy. An official document of the Italian PRM Society (SIMFER). European Journal of Physical Rehabilitataion Medicine. https://doi.org/10.23736/S1973-9087.20.06256-5.

28. Guo, X., Wang, J., Hu, D., Wu, L., Gu, L., Wang, Y., et al. (2020). Survey of COVID-19 Disease among orthopaedic surgeons in Wuhan, People's Republic of China. Journal of Bone and Joint Surgery. https://doi.org/10.2106/JBJS.20.00417.

29. Ducournau, F., Arianni, M., Awwad, S., et al. (2020). COVID19: Initial experience of an international group of hand surgeons. Hand and Surgery Rehabilitation. https://doi.org/10.1016/j.hansu r.2020.04.001.

Publisher's Note Springer Nature remains neutral with regard to jurisdictional claims in published maps and institutional affiliations. 